\title{
Weighted density approximation for bonding in molecules: ring and cage polymers
}

\author{
M B Sweatman \\ Department of Chemistry, Imperial College of Science, Technology and Medicine, \\ Exhibition Road, London SW7 2AY, UK
}

\begin{abstract}
The focus of this work is the bonded contribution to the intrinsic Helmholtz free energy of molecules. A weighted density approximation (WDA) for this contribution is presented within the interaction site model (ISM) for ring and cage polymers. The resulting density functional theory (ISM/WDA) for these systems is no more complex than theories for a pure simple fluid, and much less complex than density functional approaches that treat the bonding functional exactly. The ISM/WDA bonding functional is much more accurate than either the ISM/HNC or ISM/PY bonding functionals, which are related to the reference interaction-site model (RISM)/HNC and RISM/PY integral equations respectively, for ideal ring polymers. This means that the ISM/WDA functional should generally be more accurate for most 'real' ring or cage polymer systems when any reasonable approximation for the 'excess' contribution to the intrinsic Helmholtz free energy is employed.
\end{abstract}

\section{Introduction}

Recent advances in density functional theory (DFT) for some simple (spherically symmetric, short-range interactions) systems have produced a variety of accurate yet simple functionals [1-5]. In particular, the fundamental measure functional for hard spheres [3] is both numerically quite simple and very accurate for a wide range of density distributions. Naturally, fundamental measure theory has become popular. In contrast, DFTs for general molecular systems are often inflexible, inaccurate or laborious. These limitations are hardly surprising given the complexity that arises from additional degrees of freedom including molecular conformations, orientations and heterogeneity of molecular sites. To avoid this level of complexity some DFT approaches [5-7] 'integrate-out' the internal molecular degrees of freedom to arrive at a 'coarse-grained' simple fluid description once more. Although these coarse-grained theories are often useful, by definition they cannot describe higher resolution properties of molecules (for example, atom-atom pair correlations). 
Usually, DFTs for molecular systems with site resolution can be classified into four types: (a) those that employ the Woodward approach [8,9], (b) those that employ extensions of Wertheim's thermodynamic perturbation theory (TPT) $[10,11]$, (c) those that employ an iterative Monte Carlo (MC)-DFT scheme [12], (d) or those based on the interaction-site model (ISM) of Chandler and co-workers [13, 14]. DFTs of type (a) are practically limited to either perfectly rigid molecules or freely jointed chain molecules. This is discussed in more detail in the next section. Theories of type (b) have only been applied to tangentially bonded molecules; for example, associating hard spheres that bond on their surface. Also it is not clear, except in the work of Kierlik and Rosinberg [10], that these theories yield accurate site-site pair correlation functions, an important feature for any accurate theory of the inhomogeneous fluid. Theories of type (c) are laborious, and in any case it is useful to develop a theory that does not require MC simulations to be performed. However, DFTs of type (d) do not suffer any of the above limitations. Indeed, the closely related RISM (reference interaction-site model) integral equation [15] and its extensions [16] have been popular theories for molecular liquid structure for the past three decades. Unfortunately, the density functional application of the ISM approach has received little attention. The aim of this work is to begin to address this issue. In particular, the aim is to develop an accurate, efficient and flexible ISM/DFT for ideal ring and cage polymers.

This work describes a density functional method for molecules based on the ISM approach [13] and a weighted density approximation (WDA) for the bonded contribution to the intrinsic Helmholtz free energy (called the bonding functional in this paper). A site could be either a single atom or a group of atoms in a molecule. Only the simplest formulation of this approach, the application to pure fluids of ring and cage polymers, is presented here. Ring and cage polymers have identical sites linked to form polygons and polyhedrons respectively. This bonding functional can be applied to any non-uniformity whatever, for example to adsorption phenomena at surfaces and to the test-particle limit to derive integral equations for bulk fluids, etc.

This work does not attempt to refine density functional methods for the 'excess' contribution to the intrinsic Helmholtz free energy of molecules (defined later). This will be the focus of future work. The role of the bonding contribution to the Helmholtz free energy is to provide the correlations that occur due to bonding between sites in molecules. Without this contribution molecules will tend to dissociate. So the bonding functional plays an important role whatever the density of the system. Despite the central importance of the bonding functional for modelling molecular systems there have been relatively few attempts to tackle it. Indeed, the only accurate approach to modelling general molecular systems that have arbitrary flexibility and bond lengths is, currently, via simulation; either MC, molecular dynamics or the iterative MC-DFT scheme of Yethiraj and others [12]. Yet many accurate DFTs exist for simple (unbonded) fluids. So the complexity of the bonding functional seems to present a barrier to the application of DFT to general molecular systems. Clearly, a general and relatively simple and accurate DFT for bonding has great value.

Several other theories for molecular systems have been proposed in recent decades, often in the context of the (closely related) RISM integral equation theory. RISM theory and its extensions $[15,16]$ have proved very popular for investigating a wide range of fluid systems. This is because these theories are relatively simple and yet can give reasonably accurate results for the structure of some bulk liquids, including polymers and polar solvents. Two of the most popular 'closures' for RISM theory, namely the HNC and PY closures, correspond to different bonding functionals within the ISM approach. This work shows that these bonding functionals do not satisfy the Gibbs adsorption equation, i.e. that these DFTs are thermodynamically inconsistent in this respect. In contrast the WDA bonding functional does 
satisfy the Gibbs adsorption equation and so is likely to be more accurate for adsorption studies. Apart from the HNC and PY bonding functionals only one other ISM bonding functional is known (to this author). Stillinger proposed an ISM bonding functional [17] for amphiphiles in solution based on analysing the linear response of the system to weak perturbations in the dilute amphiphile, long-wavelength limit. The result is that bonded interactions are effectively modelled by coulombic interactions.

This work compares the WDA theory with the HNC and PY theories for bonding for three ideal ring-polymer systems, namely ideal 'dumbbells' (two rigidly linked ideal sites), ideal rigid hexagons and ideal fully flexible squares. The WDA theory is found to be significantly more accurate than the other theories when theoretical results are compared to MC simulation results. This indicates that the WDA theory for bonding should generally be much more accurate than the HNC and PY theories for molecular fluids for all reasonable treatments of the excess contribution to the intrinsic Helmholtz free energy.

This paper is organized as follows. First, an alternative DFT strategy for general molecular fluids is summarized. This establishes the motivation for a more simple and flexible theory. Then the ISM approach is introduced for pure ring and cage polymers and the WDA, HNC and PY approximations for the bonding functional are discussed. Results of these theories are presented for some ideal ring-polymer fluids. The paper is concluded by a summary with indications of future applications.

Throughout the paper it is assumed that each site in a molecule is spherical. Also, the phrase 'HNC-type approximation' is used interchangeably with the phrases 'second-order truncated functional Taylor series expansion in density' and 'second-order truncated density expansion'.

\section{Theory}

\subsection{The Woodward bonding functional}

This approach considers the properties of complete molecules in terms of the molecular density, $\rho(\boldsymbol{R})$, where $\boldsymbol{R}$ represents the degrees of freedom of a molecule. For example, a molecule with $N$ spherical sites has $\boldsymbol{R}=\left\{\boldsymbol{r}_{1}, \boldsymbol{r}_{2}, \ldots, \boldsymbol{r}_{N}\right\}$, where $\boldsymbol{r}_{1}$, etc, are the positions of the $N$ sites, while for a single non-spherical particle $\boldsymbol{R}=\{\boldsymbol{r}, \boldsymbol{\theta}\}$, where $\boldsymbol{r}$ and $\boldsymbol{\theta}$ are the position and orientation vectors. The fundamental Legendre transform relation is

$$
\Omega[\psi(\boldsymbol{R})]=F[\rho(\boldsymbol{R})]-\int \mathrm{d} \boldsymbol{R} \rho(\boldsymbol{R}) \psi(\boldsymbol{R})
$$

where

$$
\psi(\boldsymbol{R})=\mu-V^{e x t}(\boldsymbol{R})
$$

and $\Omega, F, \mu$, and $V^{e x t}$ are the grand potential, intrinsic Helmholtz free energy, molecular chemical potential and external potential, respectively. Using the fundamental theorems of DFT gives the Euler-Lagrange (EL) equation for the density profile

$$
\rho(\boldsymbol{R})=\rho_{b} \exp \left(-\beta\left(V^{e x t}(\boldsymbol{R})-\mu^{e x}\right)+c^{(0)}(\boldsymbol{R})+c^{(1)}(\boldsymbol{R})\right)
$$

where $\rho_{b}$ is the bulk molecular density, $\mu^{e x}=\mu-\ln \left(\Lambda^{3} \rho_{b}\right)$ is the excess chemical potential, $\Lambda$ is an irrelevant constant and $c^{(0)}$ and $c^{(1)}$ are the singlet-direct correlation functions due to bonded and non-bonded (excess) interactions. Integrating with respect to $\rho(\boldsymbol{R})$ gives

$$
F=F^{i d}+F^{0}+F^{e x}=\beta^{-1} \int \mathrm{d} \boldsymbol{R} \rho(\boldsymbol{R})\left(\ln \left(\Lambda^{3} \rho(\boldsymbol{R})\right)-1\right)+F^{0}+F^{e x}
$$


where $F^{0}$ and $F^{e x}$, the bonded and excess intrinsic Helmholtz free energies, are the integrals of $c^{(0)}(\boldsymbol{R})$ and $c^{(1)}(\boldsymbol{R})$, respectively.

For a molecule composed of one non-spherical site only the excess term, $F^{e x}$, is required to specify the density functional. This is the approach typically employed in density functional studies of liquid crystals [18]. Generally, a solution of this problem requires the evaluation of six-dimensional integrals (five-dimensional for linear molecules which have fewer degrees of freedom) which, although much more complicated than for simple fluids, is quite feasible and an efficient method for large molecules.

Following Woodward [8], constraints on site positions can be included straightforwardly for a multi-site molecule by specifying the bonding functional, $F^{0}$, that bonds sites together:

$$
F^{0}=\int \mathrm{d} \boldsymbol{R} \rho(\boldsymbol{R}) V^{0}(\boldsymbol{R})
$$

For a fully flexible ring-polymer with fixed bond lengths $L$ we can choose

$$
\exp \left(-\beta V^{0}(\boldsymbol{R})\right)=\prod_{i=1}^{N} \delta\left(\left|\boldsymbol{r}_{i}-\boldsymbol{r}_{i-1}\right|-L\right) / 4 \pi L^{2}
$$

where $r_{0}=r_{N}$. Additional intra-molecular interactions, such as torsional interactions between next-nearest-neighbour sites, can be introduced by including additional terms in the bonding potential. The EL equation is now

$$
\begin{aligned}
\rho(\boldsymbol{R})=\rho_{b} & \exp \left(-\beta\left(V^{\text {ext }}(\boldsymbol{R})+V^{0}(\boldsymbol{R})-\mu^{e x}\right)+c^{(1)}(\boldsymbol{R})\right) \\
=\rho_{b} \prod_{i=1}^{N} \delta\left(\left|\boldsymbol{r}_{i}-\boldsymbol{r}_{i-1}\right|-L_{i}\right) & \exp \left(-\beta\left(V^{\text {ext }}(\boldsymbol{R})-\mu^{\text {ex }}\right)+c^{(1)}(\boldsymbol{R})\right) .
\end{aligned}
$$

The density for a specific site $j$ can be calculated as

$$
\rho_{j}(\boldsymbol{r})=\int \mathrm{d} \boldsymbol{R}^{\prime} \rho\left(\boldsymbol{R}^{\prime}\right) \delta\left(\boldsymbol{r}-\boldsymbol{r}_{j}^{\prime}\right)
$$

For ring and cage polymers, where each site is identical, the site density is

$\rho_{s}(\boldsymbol{r})=\rho_{s} \int \mathrm{d} \boldsymbol{R}^{\prime} \delta\left(\boldsymbol{r}-\boldsymbol{r}_{1}^{\prime}\right) \prod_{i=1}^{N} \delta\left(\left|\boldsymbol{r}_{i}^{\prime}-\boldsymbol{r}_{i-1}^{\prime}\right|-L_{i}\right) \exp \left(-\beta\left(V^{e x t}\left(\boldsymbol{R}^{\prime}\right)-\mu^{e x}\right)+c^{(1)}\left(\boldsymbol{R}^{\prime}\right)\right)$

where $\rho_{s}=N \rho_{b}$ is the bulk site density. Unfortunately, this expression requires the evaluation of $3 N$-dimensional integrals and, unlike similar expressions for freely jointed chain polymers [9], it cannot be re-expressed in terms of $N$ three-dimensional single-site propagator functions. Indeed, for molecules that have loops or are not freely jointed the $3 N$-dimensional integrals in (8) render this approach completely impracticable even for small $N$.

\subsection{The interaction site model}

Clearly, a simpler and more flexible DFT for molecular fluids is desirable, for instance a method that is no more complex than for simple fluid mixtures and can be applied to any molecular fluid whatever. By careful design it is hoped that the accompanying reduction in accuracy of such a theory will not obscure all the interesting physics associated with molecular fluids. An approach that achieves considerable simplification and yet has site resolution is described below in the context of ring and cage polymers. 
We will rewrite the fundamental DFT equations in terms of individual sites from the start. We shall focus on ring and cage polymers for simplicity. Following Chandler et al [13] we set

$$
\Omega[\psi(\boldsymbol{r})]=F[\rho(\boldsymbol{r})]-\int \mathrm{d} \boldsymbol{r} \rho(\boldsymbol{r}) \psi(\boldsymbol{r})
$$

with

$$
\psi(\boldsymbol{r})=\mu-V^{e x t}(\boldsymbol{r})
$$

where $\rho, \mu$ and $V^{\text {ext }}$ are now the site density, chemical potential and external potential. The fundamental theorems of DFT yield an Ornstein-Zernike relation

$$
\begin{aligned}
h_{0}\left(\boldsymbol{r}, \boldsymbol{r}^{\prime}\right) / \rho(\boldsymbol{r}) & +h\left(\boldsymbol{r}, \boldsymbol{r}^{\prime}\right)=c_{0}^{(2)}\left(\boldsymbol{r}, \boldsymbol{r}^{\prime}\right)+c^{(2)}\left(\boldsymbol{r}, \boldsymbol{r}^{\prime}\right) \\
& +\int \mathrm{d} \boldsymbol{r}^{\prime \prime}\left(h_{0}\left(\boldsymbol{r}, \boldsymbol{r}^{\prime \prime}\right)+\rho\left(\boldsymbol{r}^{\prime \prime}\right) h\left(\boldsymbol{r}, \boldsymbol{r}^{\prime \prime}\right)\right)\left(c_{0}^{(2)}\left(\boldsymbol{r}^{\prime \prime}, \boldsymbol{r}^{\prime}\right)+c^{(2)}\left(\boldsymbol{r}^{\prime \prime}, \boldsymbol{r}^{\prime}\right)\right) .
\end{aligned}
$$

Here, $h_{0}$ and $h$ are the intra- and inter-molecular site-site pair total correlation functions, respectively. For example, for a uniform fluid of rigid ring and cage polymers $h_{0}$ is a sum of spherical delta functions, i.e.

$$
h_{0}\left(\boldsymbol{r}, \boldsymbol{r}^{\prime \prime}\right)=\sum_{i=2}^{N} \delta\left(\left|\boldsymbol{r}-\boldsymbol{r}^{\prime \prime}\right|-L_{i}\right) / 4 \pi L_{i}^{2}
$$

where $L_{i}$ is the distance between one site and another site (labelled $i$ ) in the same rigid molecule. For flexible ring and cage polymers the normalized delta functions in this expression can be replaced by normalized smooth functions. The site-site pair direct correlation function is composed of two terms: a bonding term, $c_{0}^{(2)}$, defined by

$$
h_{0}\left(\boldsymbol{r}, \boldsymbol{r}^{\prime}\right) / \rho(\boldsymbol{r})=c_{0}^{(2)}\left(\boldsymbol{r}, \boldsymbol{r}^{\prime}\right)+\int \mathrm{d} \boldsymbol{r}^{\prime \prime} h_{0}\left(\boldsymbol{r}, \boldsymbol{r}^{\prime \prime}\right) c_{0}^{(2)}\left(\boldsymbol{r}^{\prime \prime}, \boldsymbol{r}^{\prime}\right)
$$

and the remainder, $c^{(2)}$. The pair-direct correlation function can be integrated to give the intrinsic Helmholtz free energy. Integrating once gives the EL equation

$$
\rho(\boldsymbol{r})=\rho_{b} \exp \left(-\beta\left(V^{e x t}(\boldsymbol{r})-\mu^{e x}\right)+c_{0}^{(1)}(\boldsymbol{r})+c^{(1)}(\boldsymbol{r})\right)
$$

where $\rho_{b}$ is now the bulk site density, $\mu^{e x}$ is now the excess site chemical potential and $c_{0}^{(1)}$ and $c^{(1)}$ are the integrals of $c_{0}^{(2)}$ and $c^{(2)}$, respectively. A further integration gives

$$
F=F^{i d}+F^{0}+F^{e x}=\beta^{-1} \int \mathrm{d} \boldsymbol{r} \rho(\boldsymbol{r})\left(\ln \left(\Lambda^{3} \rho(\boldsymbol{r})\right)-1\right)+F^{0}+F^{e x}
$$

where $F^{0}$ and $F^{e x}$ are the integrals of $c_{0}^{(1)}$ and $c^{(1)}$, respectively, and $\Lambda$ is the thermal de Broglie wavelength of a site. Unfortunately, unlike $F^{0}$ in (5), the bonding contribution in (16), $F^{0}$, cannot be written as an explicit functional of $\rho(\boldsymbol{r})$ in a compact form [13]. The aim of this paper is to present and compare some approximate density functionals for $F^{0}$ within the ISM representation.

One method of proceeding $[13,14]$ is to treat bonded interactions in a similar fashion to non-bonded interactions and perform a functional Taylor series expansion in density about the bulk density:

$$
\begin{aligned}
\beta\left(F^{0}[\rho]+F^{e x}\right. & {[\rho])=\beta\left(F^{0}\left(\rho_{b}\right)+F^{e x}\left(\rho_{b}\right)\right)-\int \mathrm{d} \boldsymbol{r} \Delta \rho(\boldsymbol{r})\left(c_{0}^{(1)}\left(\rho_{b}\right)+c^{(1)}\left(\rho_{b}\right)\right) } \\
& -\frac{1}{2} \iint \mathrm{d} \boldsymbol{r}_{1} \mathrm{~d} \boldsymbol{r}_{2} \Delta \rho\left(\boldsymbol{r}_{1}\right) \Delta \rho\left(\boldsymbol{r}_{2}\right)\left(c_{0}^{(2)}\left(r_{12}, \rho_{b}\right)+c^{(2)}\left(r_{12}, \rho_{b}\right)\right) \\
+ & \beta\left(\Delta F^{0}[\rho]+\Delta F^{e x}[\rho]\right)
\end{aligned}
$$


where $\Delta \rho(\boldsymbol{r})=\rho(\boldsymbol{r})-\rho_{b}, r_{12}=\left|r_{1}-r_{2}\right|$ and $\Delta F^{0}$ and $\Delta F^{e x}$ ensure that the relation is exact. With (15) we obtain the density profile

$\rho\left(\boldsymbol{r}_{1}\right)=\rho_{b} \exp \left(-\beta V^{e x t}\left(\boldsymbol{r}_{1}\right)+\int \mathrm{d} \boldsymbol{r}_{2} \Delta \rho\left(\boldsymbol{r}_{2}\right)\left(c_{0}^{(2)}\left(r_{12} ; \rho_{b}\right)+c^{(2)}\left(r_{12} ; \rho_{b}\right)\right)+B^{0}\left(\boldsymbol{r}_{1}\right)+B\left(\boldsymbol{r}_{1}\right)\right)$

where we have set $-\beta \mu_{0}^{e x}=c_{0}^{(1)}\left(\rho_{b}\right)$ and $-\beta \mu^{e x}=c^{(1)}\left(\rho_{b}\right)$, and we define the bridge functions $B^{0}(\boldsymbol{r})=-\beta \delta \Delta F^{0} / \delta \rho(\boldsymbol{r})$ and $B(\boldsymbol{r})=-\beta \Delta \delta F^{e x} / \delta \rho(\boldsymbol{r})$.

Approximations for $c_{0}^{(2)}\left(r ; \rho_{b}\right)+c^{(2)}\left(r ; \rho_{b}\right)$ can be obtained from an integral equation for the bulk fluid, i.e. by finding self-consistent solutions in the test-particle limit. In fact, the test-site limit of the DFT (18) with $B^{0}=B=0$ generates the RISM/HNC integral equation for ring and cage polymers [15]. That is, the DFT (18) with $B^{0}=B=0$ is the densityfunctional analogue of the RISM/HNC integral equation for ring and cage polymers. In this paper, equation (18) with $B^{0}=B=0$ is called the ISM/HNC/HNC approximation. This name indicates that the theory is based on the ISM approach with HNC-type approximations for both the bonding and excess functionals. Since (18) is a DFT it can be applied to any external potential or confining geometry whatever, i.e. it can be applied to situations other than the test-site limit. For example, it can be used to investigate the equilibrium properties of ring and cage polymers adsorbed onto solid surfaces, in slit pores, etc. Other approximations for $B^{0}$ and $B$ are also useful, including a PY-type approximation (ISM/PY/PY)

$\rho\left(\boldsymbol{r}_{1}\right)=\rho_{b} \exp \left(-\beta V^{e x t}\left(\boldsymbol{r}_{1}\right)\right)\left(1+\int \mathrm{d} \boldsymbol{r}_{2}\left(\rho\left(\boldsymbol{r}_{2}\right)-\rho_{b}\right)\left(c_{0}^{(2)}\left(r_{12} ; \rho_{b}\right)+c^{(2)}\left(r_{12} ; \rho_{b}\right)\right)\right)$

which is the density functional analogue of the RISM/PY integral equation closure [15].

\subsection{Weighted density approximation for bonding in ring and cage polymers}

The focus of this work is a WDA for the bonding functional of ring and cage polymers. We know that WDAs have been successful for simple fluids [1-5]. Very often a weighted density functional for a contribution to the intrinsic Helmholtz free energy functional is written as

$$
F=\int \mathrm{d} \boldsymbol{r} \rho(\boldsymbol{r}) \varphi(\bar{\rho}(\boldsymbol{r}))
$$

where $\varphi$ is the respective free energy contribution per particle of a bulk fluid and

$$
\bar{\rho}\left(\boldsymbol{r}_{1}\right)=\int \mathrm{d} \boldsymbol{r}_{2} \rho\left(\boldsymbol{r}_{2}\right) w\left(r_{12} ; \bar{\rho}\left(\boldsymbol{r}_{1}\right)\right)
$$

where $w$ is a suitable weight function. We can see that the ideal contribution to the intrinsic Helmholtz free energy of a simple fluid can be defined exactly by this recipe with $\varphi(\rho) \rightarrow \varphi_{i d}(\rho)=\beta^{-1}\left(\ln \left(\Lambda^{3} \rho\right)-1\right)$ and $w(r) \rightarrow w_{i d}(r)=\delta(r)$. Curtin and Ashcroft [19] and Tarazona [1] employed this general functional form for the excess contribution to hardsphere fluids. These hard-sphere functionals are popular and known to be quite accurate. It has also been used for the contribution arising from attractive interactions in super- [20] and sub-critical [21] Lennard-Jones (LJ) fluids with generally good accuracy for adsorption phenomena. The aim of this work is to find suitable functions within this recipe for the bonded contribution to the intrinsic Helmholtz free energy. Usually [1, 19-21], the weight function is defined by ensuring that pair-direct correlation functions are accurately reproduced by the functional in the uniform fluid limit. This corresponds to ensuring that the zeroth, first and second functional derivatives of $F$ with respect to $\rho(\boldsymbol{r})$ are accurate. Once the form of the functional is fixed in this way it should remain accurate for sufficiently small inhomogeneities. 
Equation (20) is re-written for the bonding functional as

$$
F^{0}=\int \mathrm{d} \boldsymbol{r} \rho(\boldsymbol{r}) \varphi_{0}\left(\bar{\rho}_{0}(\boldsymbol{r})\right)
$$

with

$$
\bar{\rho}_{0}\left(\boldsymbol{r}_{1}\right)=\int \mathrm{d} \boldsymbol{r}_{2} \rho\left(\boldsymbol{r}_{2}\right) w_{0}\left(r_{12} ; \bar{\rho}_{0}\left(\boldsymbol{r}_{1}\right)\right) .
$$

Differentiating (22) twice with respect to density for a uniform fluid with density $\rho_{u}$ gives [1, 19-21] (in Fourier space)

$$
\begin{gathered}
-\beta^{-1} c_{0}^{(2)}\left(k ; \rho_{u}\right)=2 \varphi_{0}^{\prime}\left(\rho_{u}\right) w_{0}\left(k ; \rho_{u}\right)+\rho_{u} \varphi_{0}^{\prime \prime}\left(\rho_{u}\right)\left(w_{0}\left(k ; \rho_{u}\right)\right)^{2} \\
+2 \rho_{u} \varphi_{0}^{\prime}\left(\rho_{u}\right) w_{0}\left(k ; \rho_{u}\right) w_{0}^{\prime}\left(k ; \rho_{u}\right)
\end{gathered}
$$

where dashes indicate differentiation with respect to density. Since we wish $F^{0}$ to generate accurate $c_{0}^{(2)}$ for a uniform fluid we equate (24) with the Fourier transformed uniform fluid limit of (14), i.e.

$$
\begin{gathered}
2 \varphi_{0}^{\prime}\left(\rho_{u}\right) w_{0}\left(k ; \rho_{u}\right)+\rho_{u} \varphi_{0}^{\prime \prime}\left(\rho_{u}\right) w_{0}^{2}\left(k ; \rho_{u}\right)+2 \rho_{u} \varphi_{0}^{\prime}\left(\rho_{u}\right) w_{0}\left(k ; \rho_{u}\right) w_{0}^{\prime}\left(k ; \rho_{u}\right) \\
=-\beta^{-1} h_{0}(k) / \rho_{u}\left(1+h_{0}(k)\right) .
\end{gathered}
$$

Solving for $\varphi_{0}$ and $w_{0}$ gives

$$
\begin{aligned}
& \varphi_{0}\left(\rho_{u}\right)=-\beta^{-1} f(k=0) \ln \left(\rho_{u}\right)+B \\
& 2 w_{0}(k)-w_{0}^{2}(k)=f(k) / f(k=0)
\end{aligned}
$$

where $f(k)=h_{0}(k) /\left(1+h_{0}(k)\right)$ and $B$ is a constant. This means that

$$
w_{0}(k)=1-\sqrt{ } 1-f(k) / f(k=0)
$$

which has the correct normalization properties $\left(w_{0}(k=0)=1\right)$. Putting $(22)-(27)$ together gives

$$
F^{0}=-\beta^{-1} \int \mathrm{d} \boldsymbol{r} \rho(\boldsymbol{r})\left(\ln \left(\left(\bar{\rho}_{0}(\boldsymbol{r})\right)^{\alpha}\right)+B\right)
$$

for the bonding functional where $\alpha=f(k=0)=(N-1) / N$ for ring and cage polymers. This WDA for bonding, ISM/WDA, in ring and cage polymers is the main result of this work.

Usually, a cut-off in the range of the weight function is desirable. In this work this cut-off range, $r_{c}$, is determined by inspection of the weight function for each system studied. The truncated weight function must then be renormalized to ensure that $w_{0}(k=0)=1$.

Using this bonding functional transforms the EL equation for the density profile, (15), into

$$
\begin{aligned}
& \rho\left(\boldsymbol{r}_{1}\right)=\rho_{b}^{1-\alpha} \bar{\rho}_{0}\left(\boldsymbol{r}_{1}\right)^{\alpha} \exp \left(-\beta\left(V^{e x t}\left(\boldsymbol{r}_{1}\right)-\mu^{e x}\right)\right. \\
&\left.+\alpha \int \mathrm{d} \boldsymbol{r}_{2}\left(\begin{array}{c}
\rho\left(\boldsymbol{r}_{2}\right) \\
\bar{\rho}_{0}\left(\boldsymbol{r}_{2}\right)
\end{array}-1\right) w_{0}\left(r_{12}\right)+c^{(1)}\left(\boldsymbol{r}_{1}\right)\right) .
\end{aligned}
$$

The important advantage of (29) over the Woodward approach [8, 9] is that it is easily solved provided we can calculate $c^{(1)}(\boldsymbol{r})$. That is, integrals in (29) are three-dimensional whatever the value of $N$, rather than $3 N$-dimensional as in (9), and so solution of (29) is relatively easy (provided calculation of $c^{(1)}(\boldsymbol{r})$ is straightforward). In addition, this route to a DFT for molecules is expected to yield accurate bulk pair correlations regardless of bond length, an attractive feature since it implies that the DFT will generally be accurate (for sufficiently small inhomogeneities). So, this ISM/WDA approach fulfils the simplicity and flexibility criteria required by this work and it is also expected to be accurate. This is a rare combination of attributes. The results section below confirms that the ISM/WDA theory is indeed quite accurate. 


\subsection{The Gibbs adsorption equation}

A desirable feature of any DFT is that it satisfies the Gibbs adsorption equation [22]. This equation describes a fundamental thermodynamic property of any system. Differentiating (10) with $V^{e x t}$ and $T$ constant gives

$$
\begin{gathered}
\partial \Omega \\
\partial \mu
\end{gathered}=\int \mathrm{d} \boldsymbol{r}\left(\begin{array}{c}
\partial \Omega \\
\partial \rho(\boldsymbol{r})
\end{array}\right)_{\mu} \begin{gathered}
\partial \rho(\boldsymbol{r}) \\
\partial \mu
\end{gathered}+\left(\begin{array}{c}
\partial F \\
\partial \mu
\end{array}\right)_{\rho(\boldsymbol{r})}-\int \mathrm{d} \boldsymbol{r} \rho(\boldsymbol{r}) .
$$

With a DFT approach the first term on the RHS of (30) is always zero due to minimization of the grand potential and, ideally, the middle term on the RHS should also be zero so that the fundamental equality

$$
\partial \Omega / \partial \mu=-N
$$

is obeyed. This requires the intrinsic Helmholtz free energy functional to be independent of the bulk chemical potential (at fixed $\rho(r), V^{e x t}(r)$ and $T$ ) or the bulk density or any other parameter dependent on the bulk chemical potential. Quite often, a DFT is proposed that does not satisfy this relation. Such functionals are likely to lose accuracy as adsorption increases [22]. They are also thermodynamically inconsistent in the sense that two routes for calculating the grand potential, namely (1) by integrating (31) and (2) by direct calculation from the density functional with the equilibrium density profile, will disagree [22]. And the discrepancy will grow with increasing adsorption.

Because of its HNC-type approximation for $F^{0}$, the ISM/HNC functional is dependent on the bulk density and so does not satisfy the Gibbs adsorption equation and is likely to be a poor choice for adsorption studies. The same holds for the ISM/PY functional. Since the ISM/WDA functional avoids reference to the bulk density it does satisfy the Gibbs adsorption equation and so is likely to be more accurate for adsorption studies.

Also, when $V^{\text {ext }}$ varies slowly on the length scale of the polymer we have $\bar{\rho}_{0}(\boldsymbol{r}) \approx \rho(\boldsymbol{r})$ which with (29) gives for the ISM/WDA theory

$$
\rho(\boldsymbol{r}) \approx \rho_{b}\left(\exp \left(-\beta\left(V^{e x t}(\boldsymbol{r})-\mu^{e x}\right)+c^{(1)}(\boldsymbol{r})\right)\right)^{N}
$$

which becomes exact in the limit of a uniform external potential. In contrast, the corresponding result for the ISM/HNC theory is

$\rho\left(\boldsymbol{r}_{1}\right) \approx \rho_{b} \exp \left(-\beta\left(V^{e x t}\left(\boldsymbol{r}_{1}\right)-\mu^{e x}\right)+\int \mathrm{d} \boldsymbol{r}_{2}\left(\rho\left(\boldsymbol{r}_{2}\right)-\rho_{b}\right) c_{0}^{(2)}\left(r_{12} ; \rho_{b}\right)+c^{(1)}\left(\boldsymbol{r}_{1}\right)\right)$

which does not become exact in the limit of a uniform external potential. The ISM/PY functional also fails to be exact in this limit.

These reasons, i.e. that the ISM/WDA functional is exact in the uniform fluid limit (by this I mean a fluid with any uniform density not necessarily equal to the bulk or reservoir density) and satisfies the Gibbs adsorption equation are two strong reasons for favouring it over other ISM bonding functionals that do not have these properties. We will see below that the ISM/WDA functional is also accurate for some non-uniform fluids.

\section{Results for ideal ring polymers}

Since the focus of this work is on the bonding functional, not the excess functional, emphasis is placed on results for ideal molecules. Results are obtained for ideal dumbbells, ideal rigid hexagons and ideal flexible squares. MC simulation results are compared against ISM results 
with the WDA, HNC and PY approximations for the bonding functional for each system. Results are calculated for each system adjacent to a hard wall:

$$
V_{H W}^{e x t}(z)= \begin{cases}0 ; & z \geqslant 0 \\ \infty ; & z<0\end{cases}
$$

and a LJ 9-3 wall

$$
V_{L J}^{e x t}(z)= \begin{cases}\varepsilon\left(\underset{15}{2}(\sigma / z)^{9}-(\sigma / z)^{3}\right) ; & z \geqslant 0 \\ \infty ; & z<0\end{cases}
$$

for several values of reduced temperature, $T^{*}=k_{B} T / \varepsilon$, and reduced bond length $L^{*}=L / \sigma$. Here, $z$ is the distance of a site from the wall. These results give a general indication of the accuracy of each theory for adsorption in slit pores, spherical cavities, on stationary test molecules, etc.

For each theory the required input data is the bulk fluid intra-molecular contribution to the total site-site pair correlation function, $h_{0}(r)$. For rigid dumbbells and hexagons this is easily obtained from (13). For flexible squares this function is determined in the appendix. The MC simulations of flexible square polymers involve 'folding' moves as well as positional and orientational moves. A folding move involves an attempt to re-assign the folding angles at random from an appropriate distribution (see the appendix for details). Results for $\rho(r) / \rho_{b}$, which is independent of the bulk density, are calculated for various $L^{*}$ and $T^{*}$.

\subsection{Numerical details}

The weighted densities are 3D convolutions of weight functions and density profiles. For each application below the weight functions resulting from the ISM/WDA have components similar to delta functions at $r=L$ so these convolutions are best solved by 3D fast Fourier transforms (FFTs). This requires the density profile to be spherically symmetric. Adsorption against a planar wall is isomorphic with adsorption on a spherical particle with radius $R$ in the limit $R \rightarrow \infty$. This fact allows each planar DFT problem to be solved quite easily using 3D FFTs. The error reduces with increasing $R$. We find that setting $R=100 \sigma$ produces errors of about $1 \%$ compared to solutions with very large $R$. The numerical mesh has a radial range of over $320 \sigma$ with resolution equal to $0.02 \sigma$.

Each grand canonical ensemble MC simulation is performed in a slit with width $20 \sigma$. Each wall is identical and results are obtained by averaging the results for adsorption of each site against each wall. The external potential exerted by each wall is set to zero for $z \geqslant 10 \sigma$. This slit width is sufficiently large that the results described below correspond to adsorption on a single wall.

\subsection{Dumbbells}

Figure 1(a) compares the weight function, $w_{0}(r)(27)$, and the bulk fluid site-site pair-direct correlation function, $c_{0}^{(2)}(r)(14)$, for ideal dumbbells over the range $1.5 L<r<5 L$. Each function has been scaled by $(r / L)^{2}$ so that a true reflection of the significance of each function is apparent. A cut-off at $r_{c}=3 L$ is chosen for each theory. That is, $w_{0}$ entering the WDA functional and $c_{0}^{(2)}$ entering the HNC and PY-type functionals are cut at $r_{c}=3 L$ and then re-scaled to ensure that the values of $w_{0}(k=0)$ and $c_{0}^{(2)}(k=0)$ are correct $(1$ and $(N-1) / N$ respectively).

Figure 1(b) shows $\rho(r) / \rho_{b}$ predicted by each theory for ideal dumbbells against a hard wall and a 9-3 LJ wall. Each 9-3 LJ wall result is shifted vertically in this figure for clarity. 

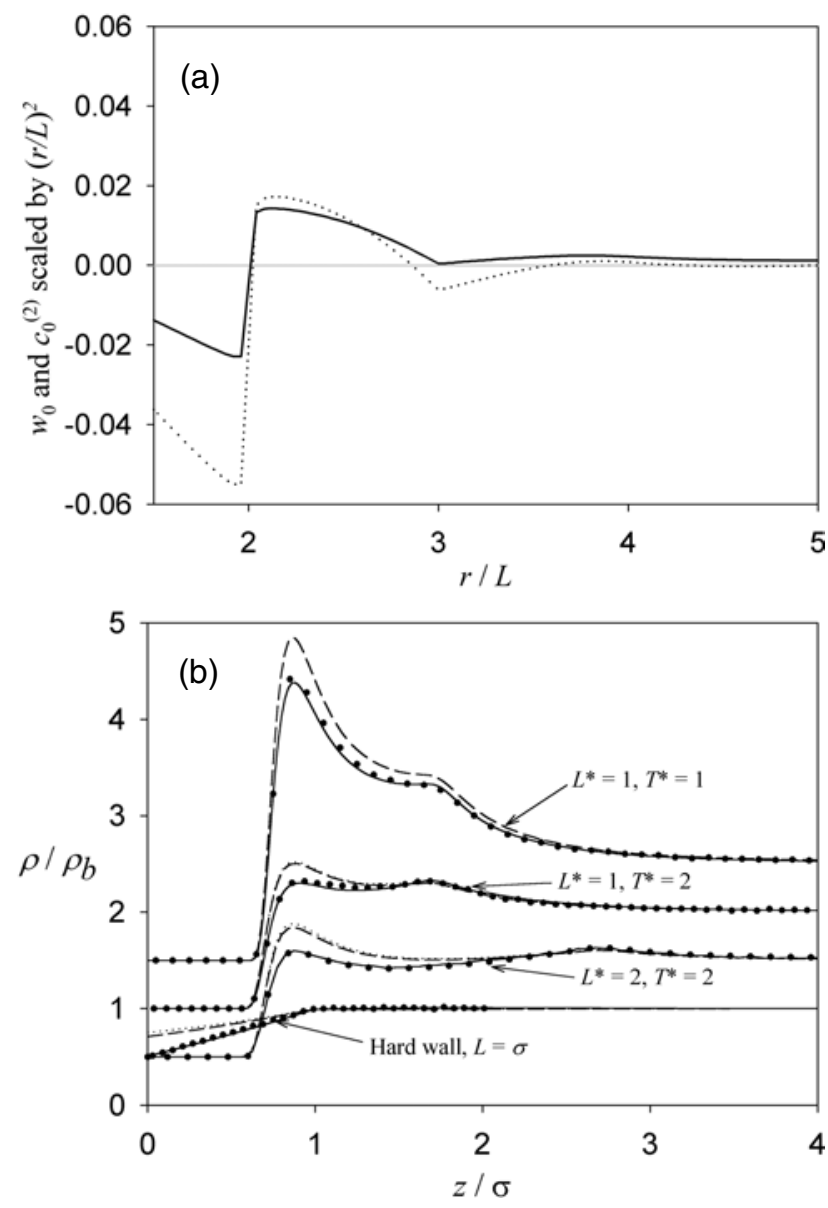

Figure 1. (a) Weight function entering the WDA for bonding, $w_{0}(r)$ (full curve), and bulk fluid site-site pair-direct correlation function entering the HNC and PY-type bonding functionals, $c_{0}^{(2)}(r)$ (dotted curve), for ideal dumbbells with bond length $L$. Each function is scaled by $(r / L)^{2}$ and, due to 'ringing' caused by the finite mesh, the lines are drawn through every second data point only. (b) Relative site densities, $\rho(z) / \rho_{b}$, for an ideal dumbbell fluid adsorbed by hard and 9-3 LJ walls. Full, dotted and broken curves correspond to WDA, HNC and PY functionals for bonding while the circles are MC simulation results.

We see that the WDA bonding functional is very accurate for each $L$ and $T$ studied. Since the WDA bonding functional yields the correct bulk fluid equation of state it also has the correct hard wall contact density. However, the HNC and PY-type results are quite inaccurate very close to each wall and they do not predict the correct bulk fluid equation of state. Indeed, for $L^{*}=1.0$ and $T^{*}=1.0$ the ISM/HNC theory has no solution. Note that each theory, when solutions exist, is able to predict the 'hump' in $\rho(r) / \rho_{b}$ that occurs at $r^{*} \approx 1+L^{*}$. Since dumbbells can be viewed as freely jointed chain polymers exact results for this system can be obtained from the Woodward approach ((9) with $N=2)$ with little additional difficulty.

\subsection{Stiff hexagons}

Figure 2(a) compares the weight function, $w_{0}(r)$, and the bulk fluid site-site pair-direct correlation function, $c_{0}^{(2)}(r)$, for stiff ideal hexagons over the range $2.5 L<r<8 L$. A cut-off of $r_{c}=5.26 \mathrm{~L}$ is chosen for each theory. 

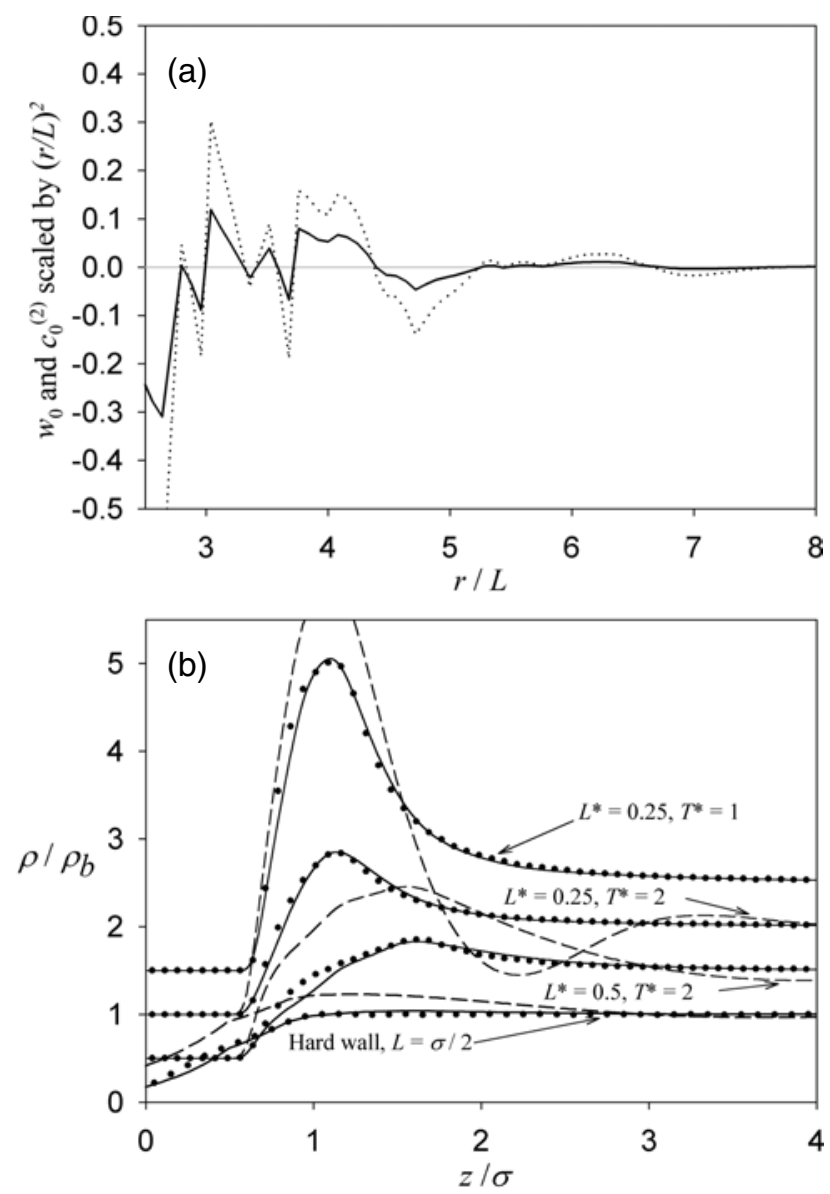

Figure 2. (a) As for figure 1(a) except for the ideal stiff hexagonal polymer fluid. (b) As for figure 1(b) except for the ideal stiff hexagonal polymer fluid.

Figure 2(b) shows $\rho(r) / \rho_{b}$ predicted by each theory for stiff ideal hexagons against a hard wall and a 9-3 LJ wall. We see that the WDA bonding functional is quite accurate for each $L$ and $T$ studied although significant errors can be seen very close to each wall. The PY-type results are completely inaccurate and the ISM/HNC theory has no solution for any of these states. The errors in these WDA results are much larger than for ideal dumbbells. This might be due to the combination of increased orientational order of ideal hexagons relative to ideal dumbbells when close to an adsorbing wall and the lack of explicit orientational degrees of freedom in the ISM DFTs. As these hexagons are stiff exact results could also be obtained from (3) with $\boldsymbol{R}$ representing positional and orientational degrees of freedom.

\subsection{Flexible squares}

Figure 3(a) compares the weight function, $w_{0}(r)$, and the bulk fluid site-site pair-direct correlation function, $c_{0}^{(2)}(r)$, for flexible ideal square polymers over the range $1.5 L<r<7 L$. A cut-off of $r_{c}=4.6 \mathrm{~L}$ is chosen for each theory.

Figure 3(b) shows $\rho(r) / \rho_{b}$ predicted by each theory for flexible ideal square polymers against a hard wall and a 9-3 LJ wall. We see that the WDA bonding functional has good 

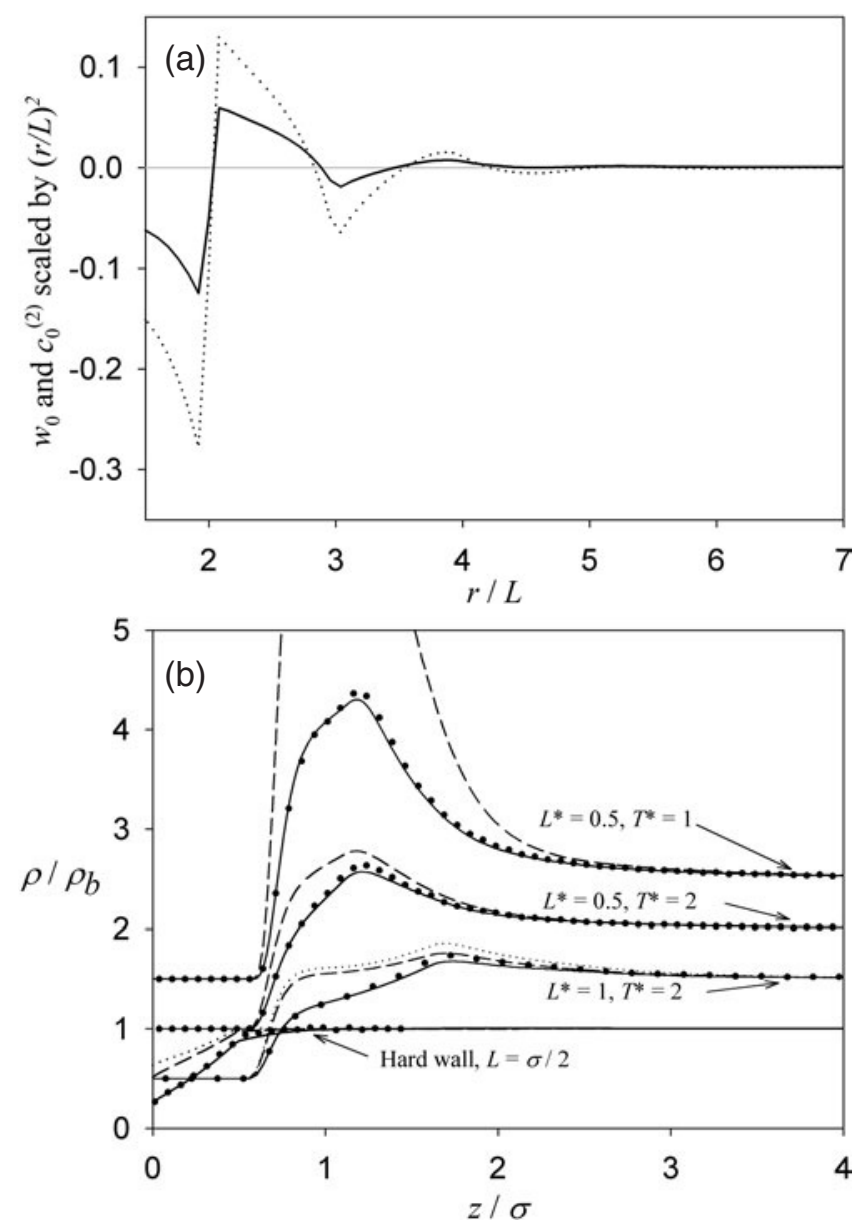

Figure 3. (a) As for figure 1(a) except for the ideal flexible square polymer fluid. (b) As for figure 1(b) except for the ideal flexible square polymer fluid.

accuracy for each $L$ and $T$ studied, with the largest errors occurring at the maximum of each profile. The HNC and PY-type results are poor when solutions exist. The errors in these WDA results are slightly larger than for ideal dumbbells and much better than for stiff ideal hexagons. Once again, this might be due to the increased orientational order of ideal hexagons relative to both ideal dumbbells and flexible squares when close to an adsorbing wall.

This application to ideal flexible square polymers clearly demonstrates the utility of this WDA approach. Flexible square polymers have eight degrees of freedom. Yet the ISM DFTs exhibit just three degrees of freedom. No other DFT in the literature has this combination of simplicity and accuracy.

\section{Summary}

A WDA for the bonded contribution to the Helmholtz free energy of ring and cage polymers has been developed and applied successfully to calculate distribution functions for some ideal ring polymers. The results of the previous section indicate that the WDA bonding functional 
is much more accurate than the HNC and PY bonding functionals for molecular studies, regardless of the size or flexibility of the molecules. This is to be expected given that the WDA bonding functional satisfies the Gibbs adsorption equation and gives the exact contribution to the Helmholtz free energy for any uniform fluid whereas the HNC and PY-type theories fail in this respect. As predicted, the performance of the HNC and PY bonding functionals deteriorates with increasing adsorption.

It follows that we can expect the WDA functional to generally be much more accurate than the HNC and PY functionals for $F^{0}$ even when $F^{e x}$ is not zero, i.e. for real molecules (provided the same functional for $F^{e x}$ is used when comparing these approaches). So the accuracy of the RISM integral equation $[15,16]$, whether in the test-site limit or the 3D test-molecule limit, can be improved by replacing HNC or PY bonding functionals with the WDA bonding functional. The additional complexity of the WDA bonding functional relative to the HNC or PY bonding functionals is not particularly significant.

The WDA bonding functional is also relatively simple and flexible. It is much simpler than the Woodward prescription for molecular DFTs $[8,9]$ described earlier for non-uniform ring and cage polymer fluids, i.e. it is no more complex than theories for pure simple fluids regardless of the number of sites in, or flexibility of, the polymer. This is particularly important for modelling flexible ring polymers that have very many degrees of freedom. Within the WDA for bonding the number of degrees of freedom is always reduced to three, which means that calculations can be performed quite easily for some idealized geometries. The complexity of the real system is contained, in a limited manner, within the prescription for the bulk fluid sitesite total pair correlation function, $h_{0}(r)$. The accuracy of the ISM/WDA theory is not affected by bond length and so it is likely that it can be applied successfully to realistic molecules. Note that theories based on Wertheim's TPT1 $[10,11]$ have only ever been applied to molecules with sites that bond on their surface. Clearly, the theory is more complex than coarse-grained approaches [5-7], but these approaches do not resolve at the site level. Essentially, ISM DFTs treat molecular fluids as simple fluid site mixtures with additional bonding correlations.

The WDA bonding functional for ring and cage polymers has potential applications to systems such as diatomic nitrogen, ethane, benzene, etc, and could also be used to model methane, carbon tetrachloride, etc. For these real fluids an excess functional, $F^{e x}$, is also required, and specification of this functional will form the focus of future work. The WDA bonding functional is likely to lose accuracy as the degree of fluid inhomogeneity increases, i.e. when the density profile varies quickly over the length scale of bond lengths. It would be interesting to examine the dimensional cross-over behaviour of the ISM/WDA functional and to derive corresponding bonding functionals for systems with dimensionality other than three.

Apart from the applications to ideal ring and cage polymers described here it might be possible to extend the WDA bonding functional to more general molecules and other systems without introducing significant additional complexity. Flexible ring polymers with adjacent sites linked by harmonic interactions are particularly interesting due to the isomorphism between this classical system and Feynman's path-integral formulation of quantum mechanics for quantum particles [23]. This mapping is used routinely in path-integral MC simulations [24]. Indeed, simply modifying the flexible square model considered in this paper so that it has harmonic bonds instead of bonds with fixed length leads to a path-integral model with four 'polymer beads' of a dilute quantum gas, and the density profile in figure 3(b) would then correspond to results obtained by an approximate solution of the Schrödinger equation for particles with Boltzmann statistics. This suggests that some fluid problems with significant quantum behaviour could be solved (approximately) by use of a classical DFT [25]. This approach has already been used to model freezing of quantum fluids [26] but has not been extended to more general fluid properties. Extension of the WDA bonding functional to 


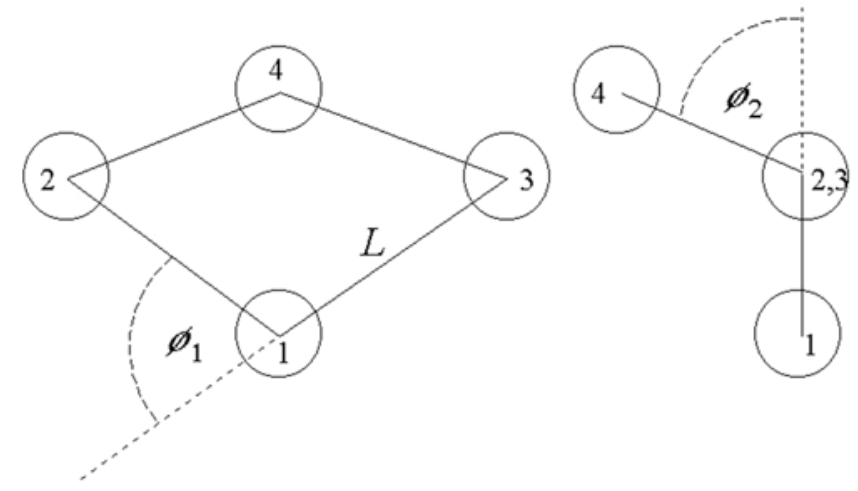

Figure A.1. Schematic diagram of a flexible square polymer. $L$ is the bond length while $\phi_{1}$ and $\phi_{2}$ are the folding angles.

molecules with non-identical sites, e.g. carbon dioxide, alkanes, polymers, amphiphiles, etc, is also desirable.

\section{Acknowledgment}

I am grateful to Fernando Bresme for some stimulating discussions.

\section{Appendix}

Consider a flexible square polymer with arbitrary configuration. It has eight degrees of freedom - three for its position, $r$, and three for its orientation, $\theta$, relative to a fixed frame of reference and two more for its 'folding angles', denoted $\phi_{1}$ and $\phi_{2}$ in figure A.1. The configurational integral for a single ideal flexible square polymer can be written as

$Z=\int \mathrm{d} \boldsymbol{r} \int \mathrm{d} \boldsymbol{\theta} \int_{0}^{\pi} \mathrm{d} \phi_{1} P\left(\phi_{1}\right) \int_{0}^{2 \pi} \mathrm{d} \phi_{2} P\left(\phi_{2}\right) \exp \left(-\beta V^{e x t}\left(\boldsymbol{r}, \boldsymbol{\theta}, \phi_{1}, \phi_{2}\right)\right)$

where

$$
\begin{aligned}
& P\left(\phi_{1}\right)={ }_{4}^{3} \sin \left(\phi_{1}\right) \sin \left(\phi_{1} / 2\right) \\
& P\left(\phi_{2}\right)=1 / 2 \pi .
\end{aligned}
$$

For the uniform fluid the probability distribution for site 4 to be at a distance $r$ from point 1 (see figure A.1) can be written as

$$
P(r)=\int_{0}^{\pi} \mathrm{d} \phi_{1}{ }_{4}^{3} \sin \left(\phi_{1}\right) \sin \left(\phi_{1} / 2\right) \int_{0}^{2 \pi}{ }_{2 \pi}^{\mathrm{d} \phi_{2}} \delta\left(r-d^{\prime}\left(\phi_{1}, \phi_{2}\right)\right)
$$

where

$$
d^{\prime}=L \sin \left(\phi_{1} / 2\right) \sqrt{ } 2\left(1+\cos \left(\phi_{2}\right)\right)
$$

Evaluating the integral (38) gives

$$
P(r)={ }_{4 \pi}^{3}(2 \sqrt{ } 1-a+a \ln ((2-a+2 \sqrt{ } 1-a) / a))
$$

where $a=(r / 2 L)^{2}$. This means that for a flexible square polymer we must have

$h_{0}(r)=2 \delta(L-r) / 4 \pi L^{2}+\underset{(4 \pi r)^{2}}{3}(2 \sqrt{ } 1-a+a \ln ((2-a+2 \sqrt{ } 1-a) / a))$. 
A folding move in a MC simulation attempts to reassign the folding angles, $\phi_{1}$ and $\phi_{2}$, of a particular square polymer. Since the probability distribution for $\phi_{2}$ is uniform, $\phi_{2}$ can be chosen at random with uniform probability from the interval $\{0,2 \pi\}$. But since the probability distribution for $\phi_{1}$ is non-uniform we instead choose a dummy parameter, $x$, at random with uniform probability from the interval $\{0,1\}$ and then choose $\phi_{1}$ such that

$$
x=\int_{0}^{\phi_{1}} \mathrm{~d} \phi_{1}^{\prime} P\left(\phi_{1}^{\prime}\right)
$$

i.e. we choose $\phi_{1}=2 \sin ^{-1}\left(x^{1 / 3}\right)$.

\section{References}

[1] Tarazona P 1985 Phys. Rev. A 312672 Tarazona P 1985 Phys. Rev. A 323148

[2] Rosenfeld Y 1989 Phys. Rev. Lett. 63980

Phan S, Kierlik E, Rosinberg M L, Bildstein B and Kahl G 1993 Phys. Rev. E 48618

Kierlik E and Rosinberg M L 1990 Phys. Rev. A 423382

[3] Tarazona P 2000 Phys. Rev. Lett. 84694

[4] Schmidt M 2000 J. Phys.: Condens. Matter 1110163

[5] Sweatman M B 2002 J. Phys.: Condens. Matter 1411921

[6] Schmidt M, Löwen H, Brader J H and Evans R 2000 Phys. Rev. Lett. 851934

[7] Groh B and Schmidt M 2001 J. Chem. Phys. 1145450

[8] Woodward C E 1991 J. Chem. Phys. 943183

[9] For example, see Frischknecht A L, Curro J G and Frink L J D 2002 J. Chem. Phys. 11710398 Yu Y X and Wu J 2002 J. Chem. Phys. 1167094

[10] Kierlik E and Rosinberg M L 1992 J. Chem. Phys. 979222

Kierlik E and Rosinberg M L 1993 J. Chem. Phys. 993950

Kierlik E and Rosinberg M L 1994 J. Chem. Phys. 1001716

[11] For example, also see

Segura C J, Chapman W G and Shukla K P 1997 Mol. Phys. 90759

Yu Y X and Wu J 2002 J. Chem. Phys. 1172368

[12] For example, see

Muller M and MacDowell L G 2000 Macromolecules 333902

Hooper J B, McCoy J D and Curro J G 2000 J. Chem. Phys. 1123090

Yethiraj A, Fynewever H and Shew C Y 2001 J. Chem. Phys. 1144323

[13] Chandler D, McCoy J D and Singer S J 1986 J. Chem. Phys. 855971

Chandler D, McCoy J D and Singer S J 1986 J. Chem. Phys. 855977

[14] See also

Chandler D, McCoy J D and Singer S J 1987 J. Chem. Phys. 874853

Ding K, Chandler D, Smithline S J and Haymet A D J 1987 Phys. Rev. Lett. 591698

McMullen W E and Freed K F 1990 J. Chem. Phys. 921413

McMullen W E and Freed K F 1990 J. Chem. Phys. 939130

[15] Chandler D and Anderson H C 1972 J. Chem. Phys. 571930

Hansen J-P and MacDonald I R 1986 Theory of Simple Liquids 2nd edn (London: Academic)

Gray C G and Gubbins K E 1984 Theory of Molecular Fluids Volume I: Fundamentals (Oxford: Clarendon)

[16] Hirata F and Rossky P J 1981 Chem. Phys. Lett. 83329

Schweizer K S and Curro J G 1987 Phys. Rev. Lett. 58246

Cortis C M, Rossky P J and Friesner R A 1997 J. Chem. Phys. 1076400

Pickett G T and Schweizer K S 1999 J. Chem. Phys. 1106597

[17] Stillinger F 1983 J. Chem. Phys. 784654

[18] For example, see

Ginzburg V V, Glaser M A and Clark N A 1997 Liq. Cryst. 23227

Cinacchi G and Schmid F 2002 J. Phys.: Condens. Matter 1412223

[19] Curtin W A and Ashcroft N W 1985 Phys. Rev. A 322909

[20] Sweatman M B 2001 Phys. Rev. E 63031102 
[21] Sweatman M B 2002 Phys. Rev. E 65011102

[22] Sweatman M B 2000 Mol. Phys. 98573

[23] Feynman R P and Hibbs A R 1965 Quantum Mechanics and Path Integrals (New York: McGraw-Hill)

[24] For example, see

Wang Q, Johnson J K and Broughton J Q 1997 J. Chem. Phys. 1075108

[25] Chandler D and Wolynes P G 1981 J. Chem. Phys. 744078

[26] See, for example

McCoy J D, Rick S W and Haymet A D J 1990 J. Chem. Phys. 923034

Rick S W, McCoy J D and Haymet A D J 1990 J. Chem. Phys. 923040 